\title{
Hybrid function projective synchronization in discrete dynamical networks via adaptive control
}

\author{
Ghada Al-mahbashi*, M. S. Md Noorani, Sakhinah Abu Bakar \\ School of mathematical Sciences, Faculty of Science and Technology, Universiti Kebangsaan Malaysia, 43600 UKM, Selangor Darul \\ Ehsan, Malaysia.
}

\begin{abstract}
In this paper, we study the hybrid function projective synchronization between coupled complex discrete networks with different dimensions. The hybrid function projective synchronization is achieved by designing an adaptive control method. Based on the designed controller and the Lyapunov stability theory, we derive sufficient conditions to realize the hybrid function projective synchronization with different nodes. Moreover, with the adaptive update law, an adaptive control gains are obtained. Furthermore, we examine different cases of outer coupling matrix of node dynamics. Finally, we provide numerical examples to show the effectiveness of the proposed control scheme. (C)2017 All rights reserved.
\end{abstract}

Keywords: Hybrid function projective synchronization, delay coupling and non-delay coupling, discrete complex dynamical networks, adaptive control.

2010 MSC: 93C40, 34D06.

\section{Introduction}

During the last decades, synchronization of the dynamical systems has appeared as an active research area in dynamical systems. Many researchers have studied this issue theoretically and numerically because of its many applications in secure communication, control theory, telecommunications, biological networks, etc. [10,14,15]. Due to complexity of chaotic behavior, different synchronization schemes have been proposed such as complete synchronization, anti synchronization, lag synchronization, projective lag synchronization, etc. $[1,3,4,7,8,11-13,17,19,20,22]$. After born different types of graph, complex networks have attracted many attention as a powerful tool to describe many complex systems [5, 6]. Two kinds of synchronization in coupled networks can be defined. The first one is the synchronization of all nodes inside a network, which is referred to as inner synchronization. The second is happening between two coupled networks, which is called as outer synchronization, which is very complicated than inner synchronization because of the diverse connections of two networks. For example, the infectious diseases, such as AIDS, mad cow disease, bird flu, were spread between two communities or networks.

\footnotetext{
${ }^{*}$ Corresponding author

Email addresses: mahbashighada@yahoo.com (Ghada Al-mahbashi), msn@ukm.my (M. S. Md Noorani), sakhinah@ukm.my (Sakhinah Abu Bakar)

doi:10.22436/jnsa.010.11.01
}

Received 2017-01-10 
It should be pointed out that most literatures on the dynamical behaviors of complex networks are concerned with continuous-time case rather than discrete-time case. In fact, discrete-time dynamics has become an important to study computer-based simulation, experimentation and it is more suitable to model digitally transmitted signals in a dynamical way. Actually, the synchronization problem of discretetime networks has received some initial research interests. Based on open plus close loop method [9], Li et al. studied outer synchronization behavior between coupled discrete-time networks with non-delay coupling, and consider when the networks have same and different connection topologies. Zhang et al. studied impulsive synchronization problem of a general complex continuous and discrete-time dynamical network with non-delay coupling and obtained sufficient condition to guarantee the synchronization [21]. In [16], Sun et al. proposed synchronization in delay discrete-time complex networks and investigated the synchronization including inner synchronization within each network and outer synchronization between two networks by using linear matrix inequalities and investigate several criteria of synchronization stability. In [18], Wu proposed synchronization of discrete dynamical networks with non-delay and delay coupling, based on Lyapunov function methods and linear matrix inequalities. Recently, Al-mahbashi et al. [2] studied hybrid function projective synchronization with delay coupling and different dimensions in discrete dynamical networks (DCDNs) model based on Lyapunov stability theory, which this paper is a continuation of it.

Motivated by the above discussion, in this paper a hybrid function projective synchronization (HFPS) behavior of uncertain discrete complex dynamical networks (DCDNs) model with different nodes and with both non-delay and delay coupling will be considered. Actually, in this paper the proposed synchronization scheme is achieved by both ways which are reduced order and increased order in presence of different cases of topological structure of the coupled networks.

The rest of this paper is organized as follows. Section 2 introduces the discrete dynamical network model with delay coupling. A general method of HFPS by adaptive control method and Lyapunov stability theory is discussed in Section 3. Examples and their simulations are obtained in Section 4. Finally, the conclusions are drawn in Section 5.

\section{Model description}

We consider a controlled discrete complex dynamical network model with delay and non-delay coupling consisting of $\mathrm{N}$ different nodes which is described as follows:

$$
y_{i}(k+1)=g_{i}\left(y_{i}(k)\right)+c \sum_{j=1}^{N} b_{i j} \Gamma y_{j}(k)+c \sum_{j=1}^{N} b_{i j} \Gamma y_{j}(k-\tau)+u_{i}(k),
$$

where $y_{i}(k)=\left(y_{i 1}(k), y_{i 2}(k), \ldots, y_{i n}(k)\right)^{\top} \in \mathbf{R}^{n}$ denotes the state vector of the $i$-th node, $g_{i}: \mathbf{R}^{n} \longrightarrow \mathbf{R}^{n}$ is the function matrices determining the dynamic behavior of the node, $u_{i} \in \mathbf{R}^{n}$ is the control input, $c$ is the coupling strength and $\tau \geqslant 0$ is the coupling delay. Here $\Gamma=\operatorname{diag}\left(\gamma_{1}, \gamma_{2}, \ldots, \gamma_{n}\right)$ is the inner coupling matrix with $\gamma_{i}=1$ for the $i$-th state variable, i.e. matrix $\Gamma$ determines the variables with which the nodes in system are coupled. $B=\left(b_{i j} \in \mathbf{R}^{N \times N}\right)$ is the outer coupling matrix representing the topological structure of the network, where $b_{i j}$ are defined as follows: if there is a connection from node $i$ to node $j(j \neq i)$, then the coupling $b_{i j} \neq 0$; otherwise, $b_{i j}=0(j \neq i)$, and the diagonal elements of matrices $B$ are defined as

$$
b_{i i}=-\sum_{j=1, j \neq i}^{N} b_{i j}, \quad i=1,2, \ldots, N .
$$

On the other hand, the reference network (drive network) is described as follows:

$$
x_{i}(k+1)=f_{i}\left(x_{i}(k)\right)+c \sum_{j=1}^{N} a_{i j} \Gamma x_{j}(k)+c \sum_{j=1}^{N} a_{i j} \Gamma x_{j}(k-\tau),
$$


where $x_{i}(k)=\left(x_{i 1}(k), x_{i 2}(k), \ldots, x_{i m}(k)\right)^{\top} \in \mathbf{R}^{m}$ denotes the state vector of the drive system, and $f_{i}$ : $\mathbf{R}^{\mathrm{m}} \longrightarrow \mathbf{R}^{\mathrm{m}}, \mathrm{a}_{i j}$ have the same meaning as $b_{i j}$ in (2.1). When order $\mathrm{n}=\mathrm{m}$ the synchronization problem has been well studied.

Definition 2.1. The drive and the response discrete dynamical networks (2.2) and (2.1) are said to be hybrid function projective synchronization (HFPS) when $m=n$ if there exists a diagonal scaling function matrix $\mathrm{H}$ such that

$$
\lim _{k \rightarrow \infty}|| e_{i}(k)\left|=\lim _{k \rightarrow \infty}\right|\left|y_{i}(k)-H x_{i}(k)\right|=0 .
$$

In this paper, we discuss the HFPS with different dimension nodes where $n>m$ and $n<m$. When the order of the response nodes oscillators are lower than that of the order of drive nodes, the synchronization is attained in reduce-order. We can divide the drive network into two parts

$$
x_{i_{s}}(k+1)=f_{i_{s}}\left(x_{i_{s}}(k)\right)+c \sum_{j=1}^{N} a_{i j} \Gamma_{s} x_{j_{s}}(k)+c \sum_{j=1}^{N} a_{i j} \Gamma_{s} x_{j_{s}}(k-\tau),
$$

where $x_{i_{s}} \in \mathbf{R}^{n}, f_{i_{s}}: \mathbf{R}^{m} \longrightarrow \mathbf{R}^{n}$ and $\Gamma_{s}=\operatorname{diag}\left(\gamma_{s_{1}}, \gamma_{s_{2}}, \ldots, \gamma_{s_{n}}\right)$. The rest:

$$
x_{i_{r}}(k+1)=f_{i_{r}}\left(x_{i_{r}}(k)\right)+c \sum_{j=1}^{N} a_{i j} \Gamma_{r} x_{j_{r}}(k)+c \sum_{j=1}^{N} a_{i j} \Gamma_{r} x_{j_{r}}(k-\tau),
$$

where $x_{i_{r}} \in \mathbf{R}^{w}, f_{i_{r}}: \mathbf{R}^{m} \longrightarrow \mathbf{R}^{w}$ and $\Gamma_{r}=\operatorname{diag}\left(\gamma_{r_{1}}, \gamma_{r_{2}}, \ldots, \gamma_{r_{w}}\right)$. Where the orders $n, w$ satisfy $n+$ $w=\mathrm{m}$. With a suitable controller, the reduced-order hybrid function projective synchronization (HFPS) between the response network (2.1) and the drive network (2.3) can be achieved, i.e.,

$$
\lim _{k \longrightarrow \infty}|| e_{i}(k)\left|=\lim _{k \longrightarrow \infty}\right|\left|y_{i}(k)-H x_{i_{s}}(k)\right|=0,
$$

where $H=\operatorname{diag}\left(h_{1}, h_{2}, \ldots, h_{n}\right)$ is a diagonal scaling function matrix.

The second case, when the order of the drive nodes oscillator are lower than that of the order of response nodes, the synchronization is attained in increased-order. Hence, the drive network is rewritten as

$$
X i(k+1)=f_{i}\left(X_{i}(k)\right)+c \sum_{j=1}^{N} a_{i j} \Gamma_{l} X_{j}(k)+c \sum_{j=1}^{N} a_{i j} \Gamma_{l} X_{j}(k-\tau),
$$

where $X_{i}=\left(x_{i}, x_{d}\right), f_{i}\left(X_{i}(k)\right)=\left(f_{i}\left(x_{i}(k)\right), 0\right)$ and $\Gamma=\operatorname{diag}\left(\gamma_{1}, \gamma_{2}, \ldots, \gamma_{n}, \gamma_{d}\right)$ where $x_{d}, \gamma_{d} \in \mathbf{R}^{m-n}$.

In order to observe the increased-order hybrid function projective synchronization (HFPS) behavior, we define the state errors between the response network (2.1) and the drive network (2.4) as:

$$
\lim _{k \rightarrow \infty}|| e_{i}(k)\left|=\lim _{k \rightarrow \infty}\right|\left|y_{i}(k)-H X_{i}(k)\right|=0
$$

Remark 2.2. In Projective Synchronization (PS) we have a constant scaling factor. In Modified PS, the scaling factor is a matrix. In Function PS the scaling factor is functional. And finally in Modified Function PS, the scaling factor is a matrix scaling factor with functional entries.

\section{Controller design}

In this section, we prove that by using the Lyapunov stability theory, error dynamics between these two networks can be stabilized which guarantees HFPS realization. 
Theorem 3.1. Hybrid function projective synchronization between the drive network and the response network is realized for any given a diagonal scaling function matrix $\mathrm{H}$, any positive scaling factor $\alpha_{i}$, any time delay $\tau$ and any initial values $x_{i}(0), y_{i}(0)$ and $\delta_{i}(0)$ by using the following control input and adaptive laws:

$$
\begin{aligned}
u_{i}(k)= & -\delta_{i} e_{i}(k)-g_{i}\left(y_{i}(k)\right)+H\left(f_{i}\left(x_{i}(k)\right)\right)+H\left(c \sum_{j=1}^{N}\left(a_{i j}-b_{i j}\right) \Gamma x_{j}(k)\right) \\
& +H\left(c \sum_{j=1}^{N}\left(a_{i j}-b_{i j}\right) \Gamma x_{j}(k-\tau)\right) \\
\hat{\delta}_{i}(k+1)= & \delta_{i}+Q(k)\left(\hat{\delta}_{i}(k)-\delta_{i}\right)
\end{aligned}
$$

where $\hat{\delta}_{i}(k)$ is the estimation values of uncertain feedback gains $\delta_{i} . Q(k)$ is undetermined function.

Proof. Taking the dynamics error we have

$$
\begin{aligned}
e_{i}(k+1)= & y_{i}(k+1)-H x_{i}(k+1) \\
= & g_{i}\left(y_{i}(k)\right)+c \sum_{j=1}^{N} b_{i j} \Gamma e_{j}(k)+c \sum_{j=1}^{N} b_{i j} \Gamma e_{j}(k-\tau)-H\left(f_{i}\left(x_{i}(k)\right)\right)+u_{i}(k) \\
& -H\left(c \sum_{j=1}^{N}\left(a_{i j}-b_{i j}\right) \Gamma x_{j}(k)\right)-H\left(c \sum_{j=1}^{N}\left(a_{i j}-b_{i j}\right) \Gamma x_{j}(k-\tau)\right) .
\end{aligned}
$$

To show the stability of the error dynamic and the convergence of the error state to zero, we employ Lyapunov method.

Define the Lyapunov function $V(k)$ as

$$
V(k)=\sum_{i=1}^{N}\left|e_{i}(k)\right|+\sum_{i=1}^{N} \sum_{\sigma=k-\tau}^{k-1}\left|e_{i}(\sigma)\right|+\sum_{i=1}^{N} \alpha_{i}\left|\hat{\delta}_{i}(k)-\delta_{i}\right|,
$$

where $\alpha_{i}$ is any positive constant. Then we obtain:

$$
\begin{aligned}
\triangle \mathrm{V}(\mathrm{k})= & \mathrm{V}(\mathrm{k}+1)-\mathrm{V}(\mathrm{k}) \\
= & \sum_{i=1}^{N}\left|e_{i}(k+1)\right|+\sum_{i=1}^{N} \sum_{\sigma=k+1} \sum_{\tau}\left|e_{i}(\sigma)\right|+\sum_{i=1}^{N} \alpha_{i}\left|\hat{\delta}_{i}(k+1)-\delta_{i}\right| \\
& -\sum_{i=1}^{N}\left|e_{i}(k)\right|-\sum_{i=1}^{N} \sum_{\sigma=k-\tau}^{k-1}\left|e_{i}(\sigma)\right|-\sum_{i=1}^{N} \alpha_{i}\left|\hat{\delta}_{i}(k)-\delta_{i}\right| \\
= & \sum_{i=1}^{N}\left|e_{i}(k+1)\right|-\sum_{i=1}^{N}\left|e_{i}(k-\tau)\right|+\sum_{i=1}^{N} \alpha_{i}\left(\left|\hat{\delta}_{i}(k+1)-\delta_{i}\right|-\left|\hat{\delta}_{i}(k)-\delta_{i}\right|\right) .
\end{aligned}
$$

By application of the control input (3.1) to error dynamics we have

$$
\triangle \mathrm{V}(\mathrm{k})=\sum_{i=1}^{N}\left|-\delta_{i} e_{i}(k)+\sum_{i=1}^{N} b_{i j} \Gamma e_{j}(k)+\sum_{i=1}^{N} b_{i j} \Gamma e_{j}(k-\tau)\right|-\sum_{i=1}^{N}\left|e_{i}(k-\tau)\right|+\sum_{i=1}^{N} \alpha_{i}(|Q(k)|-1)\left|\left(\hat{\delta}_{i}(k)-\delta_{i}\right)\right| .
$$


Let $e(k)=\left(e_{1}(k), e_{2}(k), \ldots, e_{N}(k)\right)^{\top}, e(k-\tau)=\left(e_{1}(k-\tau), e_{2}(k-\tau), \ldots, e_{N}(k-\tau)\right)^{\top}, \delta=\left(\delta_{1}, \delta_{2}, \ldots, \delta_{N}\right)^{\top}$, $\alpha=\left(\alpha_{1}, \alpha_{2}, \ldots, \alpha_{N}\right)^{\top}$, and $P=(B \otimes \Gamma)$. Then we have

$$
\begin{aligned}
\triangle \mathrm{V}(\mathrm{k})= & |-\delta e(\mathrm{k})+\mathrm{Pe}(\mathrm{k})+\mathrm{Pe}(\mathrm{k}-\tau)|-|e(k-\tau)|+\alpha(|\mathrm{Q}(\mathrm{k})|-1)|(\hat{\delta}(\mathrm{k})-\delta)| \\
\leqslant & |\mathrm{Pe}(\mathrm{k})+\mathrm{Pe}(\mathrm{k}-\tau)-\hat{\delta}(\mathrm{k}) e(\mathrm{k})|-|e(\mathrm{k}-\tau)|+(|e(\mathrm{k})|+\alpha(|\mathrm{Q}(\mathrm{k})|-1))|\hat{\delta}(\mathrm{k})-\delta| \\
\leqslant & (|\mathrm{P}-\hat{\delta}(\mathrm{k})|-1)|e(\mathrm{k}-\tau)|+|\mathrm{P} e(\mathrm{k})+\hat{\delta}(\mathrm{k}) e(\mathrm{k}-\tau)-\hat{\delta}(\mathrm{k}) e(\mathrm{k})| \\
& +(|e(\mathrm{k})|+\alpha(|\mathrm{Q}(\mathrm{k})|-1))|\hat{\delta}(\mathrm{k})-\delta| \\
\leqslant & (|\mathrm{P}-\hat{\delta}(\mathrm{k})|-1)|e(\mathrm{k}-\tau)|-|\mathrm{P} e(\mathrm{k})+\hat{\delta}(\mathrm{k}) e(\mathrm{k}-\tau)-\hat{\delta}(\mathrm{k}) e(\mathrm{k})| \\
& +(|e(\mathrm{k})|+\alpha(|\mathrm{Q}(\mathrm{k})|-1)) \mid \hat{\delta}(\mathrm{k})-\delta) \mid \\
\leqslant & (|\mathrm{P}-\hat{\delta}(\mathrm{k})|-1)|e(\mathrm{k}-\tau)|+(|e(k)|+\alpha(|\mathrm{Q}(\mathrm{k})|-1))|\hat{\delta}(\mathrm{k})-\delta| .
\end{aligned}
$$

Obviously if

$$
|\mathrm{P}-\hat{\delta}(k)|<1
$$

and

we obtain

$$
|\mathrm{Q}(\mathrm{k})|=1-\frac{1}{\alpha}|e(k)|
$$

$$
\Delta \mathrm{V}(\mathrm{k})<0 .
$$

According to the Lyapunov stability theory the error vector $e(k)$ asymptotically converges to zero as $k \longrightarrow \infty$. So, HFPS with different dimensional is realized by using the controller (3.1) and the update law (3.2). This completes the proof.

Corollary 3.2. For any given diagonal matrix $\mathrm{H}$, if the drive network (2.2) and the response network (2.1) have same outer coupling matrices, i.e., $\mathrm{A}=\mathrm{B}$, then the two networks can achieve HFPS under the following controllers:

$$
\begin{aligned}
u_{i}(k) & =-\delta_{i} e_{i}(k)-g_{i}\left(y_{i}(k)\right)+H\left(f_{i}\left(x_{i}(k)\right)\right), \\
\hat{\delta}_{i}(k+1) & =\delta_{i}+Q(k)\left(\hat{\delta}_{\mathfrak{i}}(k)-\delta_{i}\right) .
\end{aligned}
$$

Corollary 3.3. For any given diagonal matrix $\mathrm{H}$, if the outer coupling matrix of drive network (2.2) $A=0$, then the single drive discrete system and response networks can achieve HFPS under the following controllers:

$$
\begin{aligned}
u_{i}(k) & =-\delta_{i} e_{i}(k)-g_{i}\left(y_{i}(k)\right)+H\left(f_{i}\left(x_{i}(k)\right)\right), \\
\hat{\delta}_{i}(k+1) & =\delta_{i}+Q(k)\left(\hat{\delta}_{i}(k)-\delta_{i}\right) .
\end{aligned}
$$

\section{Numerical simulations}

To verify and demonstrate the effectiveness of the proposed method, numerical examples are provided. These examples discuss hybrid function projective synchronization with different nodes and dimensions. The first simulation deals with the case when the order of the drive nodes is reduced when $m>n$. The second example examines the case when the order of the nodes dynamics of the drive system is increased when $m<n$. In each situation, different cases of outer coupling matrix are considered.

4.1. Case I: reduced-order synchronization when $m>n$

Take the Hénon-like map (4.1) as the drive network with 10 nodes which is described as follows:

$$
\begin{aligned}
& x_{i 1}(k+1)=1+x_{i 3}(k)-a x_{i 2}^{2}(k), \\
& x_{i 2}(k+1)=1+b x_{i 2}(k)-a x_{i 1}^{2}(k), \\
& x_{i 3}(k+1)=b x_{i 1}(k) .
\end{aligned}
$$

For the response network, the first 5 nodes are described by the following 2D Hénon map (4.2): 


$$
y_{i 1}(k+1)=1-\alpha y_{i 1}^{2}(k)+y_{i 2}(k), \quad y_{i 2}(k+1)=\beta y_{i 1}(k) .
$$

The other 5 nodes are described by the following 2D Lozi map (4.3):

$$
y_{i 1}(k+1)=1-r\left|y_{i 1}(k)\right|+s y_{i 2}(k), \quad y_{i 2}(k+1)=s y_{i 1}(k),
$$

where $a=1.4, b=0.2, \alpha=1.4, \beta=0.3,0<s<1,1+s<r<2-\frac{s}{2}$. We choose $r=1.7, s=0.5$.

The bifurcation diagrams of the corresponding state variables of the systems are shown in Figure 1 . We can see that, the maximum value of $x_{2}$ in Hénon-like map is 1.5, the maximum value of $y_{1}$ in $2 \mathrm{D}$ Hénon map is 1.3 and the maximum value of $y_{1}$ in Lozi map is 1.8 with respect to $b, \beta$, s being varied. Thus, the evolution regions of the network error $\left|e_{i}(k)\right|$ is $(0,1.8)$. If we take the coefficient value is $\alpha_{i}>1.8$, the undetermined function $Q(k)$ must satisfy the condition (3.4).

(a)

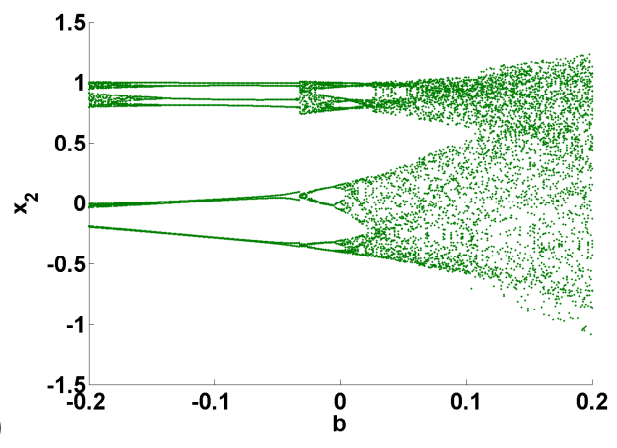

(b)

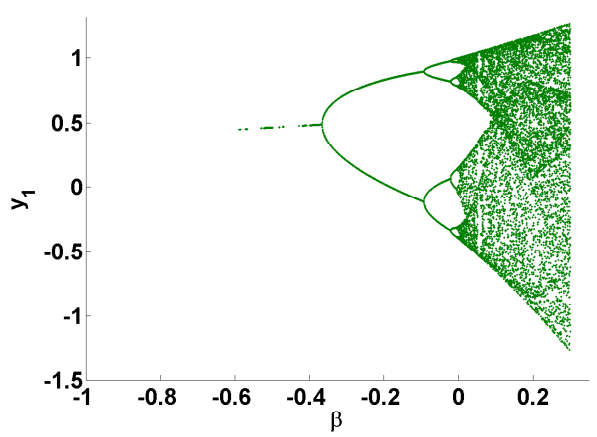

(c)

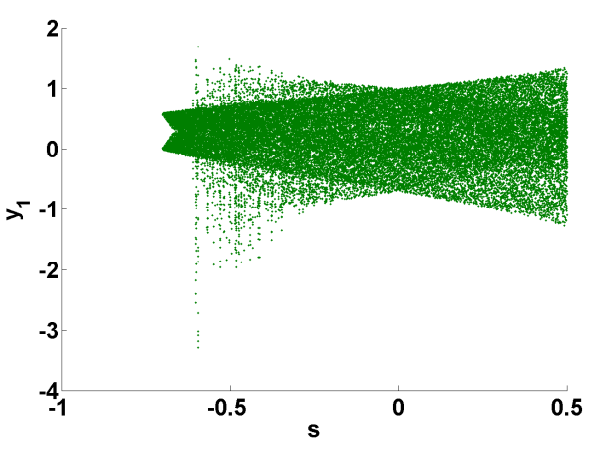

Figure 1: Bifurcation diagram of (a) Hénon-like map; (b) 2D Hénon map; (c) Lozi map.

In this simulation, initial condition of each node is chosen randomly, coupling strength is $c=0.2$, the feedback control gain is $\delta_{i}=0.45$ which can be taken arbitrarily as it satisfies the condition (3.3). The time delay coupling is $\tau=0.01, \alpha_{i}=3$, and $H=\operatorname{diag}[2 \cos (2 \pi k / 30), 0.5+\sin (\pi k / 5)]$. Inner coupling for the drive and response dynamics are assumed to be $I_{m}, I_{n}$, respectively. The outer coupling configuration matrices $A=\left(a_{i j}\right)$ and $B=\left(b_{i j}\right)$ are chosen to be as follows:

$$
A=\left(\begin{array}{cccccccccc}
-3 & 1 & 1 & 1 & 0 & 0 & 0 & 0 & 0 & 0 \\
0 & -2 & 1 & 1 & 0 & 0 & 0 & 0 & 0 & 0 \\
1 & 1 & -5 & 0 & 1 & 0 & 1 & 1 & 0 & 0 \\
0 & 0 & 1 & -3 & 1 & 1 & 0 & 0 & 0 & 0 \\
1 & 1 & 1 & 0 & -4 & 1 & 0 & 0 & 0 & 0 \\
0 & 0 & 0 & 1 & 0 & -3 & 1 & 1 & 0 & 0 \\
0 & 0 & 1 & 0 & 0 & 1 & -3 & 0 & 1 & 0 \\
0 & 1 & 0 & 1 & 0 & 0 & 1 & -3 & 0 & 0 \\
0 & 0 & 0 & 0 & 0 & 1 & 0 & 0 & -1 & 0 \\
1 & 0 & 0 & 0 & 0 & 1 & 0 & 1 & 0 & -3
\end{array}\right)
$$




$$
\mathrm{B}=\left(\begin{array}{cccccccccc}
-4 & 1 & 0 & 0 & 1 & 1 & 0 & 1 & 0 & 0 \\
0 & -2 & 0 & 1 & 0 & 1 & 0 & 0 & 0 & 0 \\
1 & 1 & -3 & 0 & 1 & 0 & 0 & 0 & 0 & 0 \\
0 & 0 & 1 & -4 & 1 & 1 & 1 & 0 & 0 & 0 \\
0 & 1 & 1 & 0 & -3 & 1 & 0 & 0 & 0 & 0 \\
0 & 0 & 1 & 1 & 0 & -3 & 0 & 1 & 0 & 0 \\
0 & 0 & 0 & 0 & 1 & 1 & -3 & 0 & 1 & 0 \\
0 & 1 & 0 & 1 & 0 & 0 & 1 & -5 & 1 & 1 \\
0 & 1 & 0 & 0 & 0 & 1 & 0 & 0 & -2 & 0 \\
1 & 0 & 1 & 0 & 1 & 0 & 0 & 0 & 1 & -4
\end{array}\right)
$$

(a)

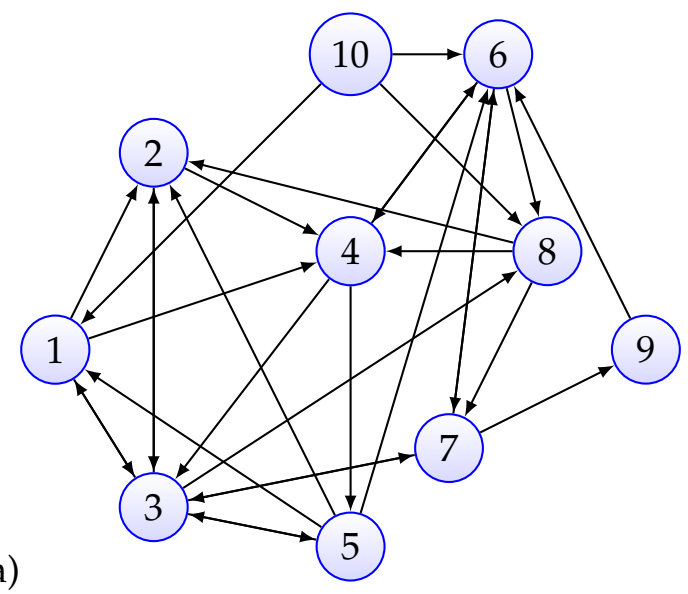

(b)

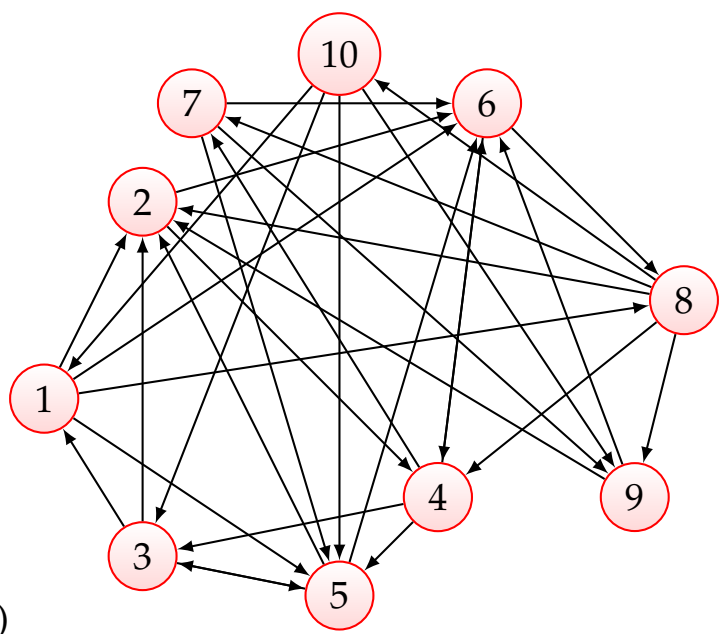

Figure 2: Network structure of non-delay and delay coupling: (a) of the drive network; (b) of the response network.

\subsubsection{The networks have different topological structures $(A \neq B)$.}

The numerical results are presented in Figures 3-5. The time evolution of the synchronization errors is depicted in Figure 3, which show that upon the activation of the control functions at $t=0.01$. This figure displays the error states converge to zero which stabilizes the error dynamic system, so HFPS between the discrete dynamical networks is realized. The estimation value of uncertain feedback gains $\hat{\delta}_{i}(i=1,2, \ldots, N)$ are depicted in Figure 4, which approach to the fixed value $\delta_{i}$, thus the identification law is effective. The state trajectories between the 7 th nodes of networks are depicted in Figure 5.

(a)

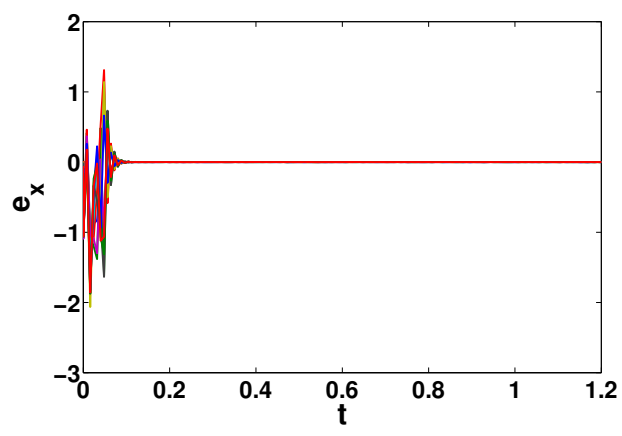

(b)

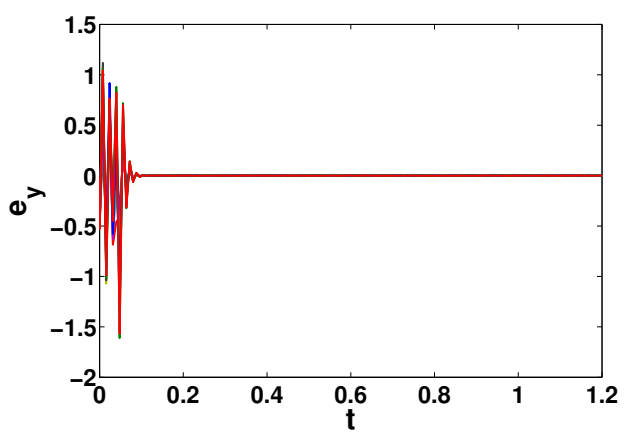

Figure 3: Dynamics of reduced-order HFPS errors with delay and non-delay coupling when $A \neq B$ : (a) errors of $x$ components; (b) errors of $y$ components. 


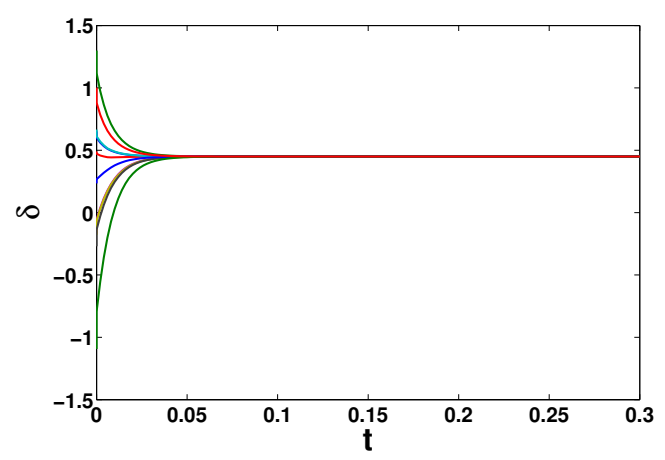

Figure 4: The estimated parameters $\hat{\delta}_{i}$ of adaptive feedback gains (3.2) in reduced-order case.

(a)

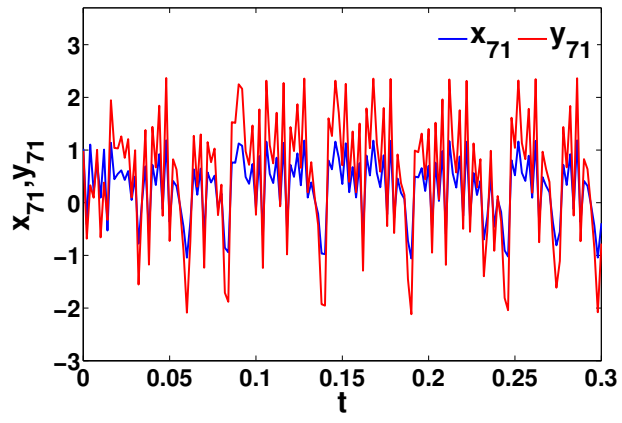

(b)

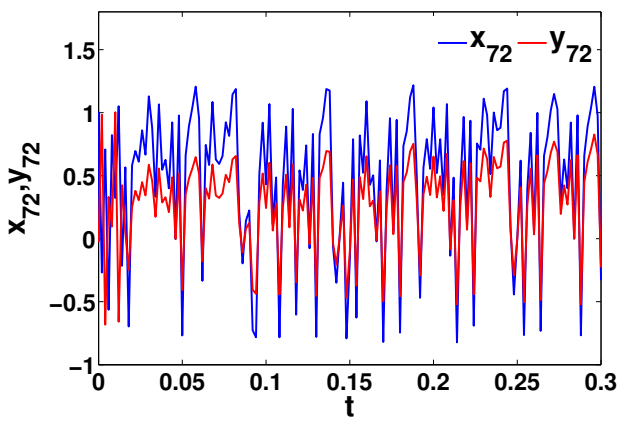

Figure 5: The time evolution of the variables of the 7th nodes of networks with delay and non-delay coupling when $A \neq B$.

\subsubsection{The networks have identical topological structure $(\mathrm{A}=\mathrm{B})$}

In this numerical results, the time evolution of the synchronization errors is illustrated in Figure 6, which shows that upon the activation of the control functions at $t=0.01$. The identified parameters of the adaptive gains are depicted in Figure 7 which converge to the fixed values $\delta_{i}$. These results prove that the required synchronization has been achieved with our designed control law (3.5)-(3.6) and under the conditions (3.3), (3.4). Figure 8 depicts the state trajectories between the 4 th nodes in networks.

(a)

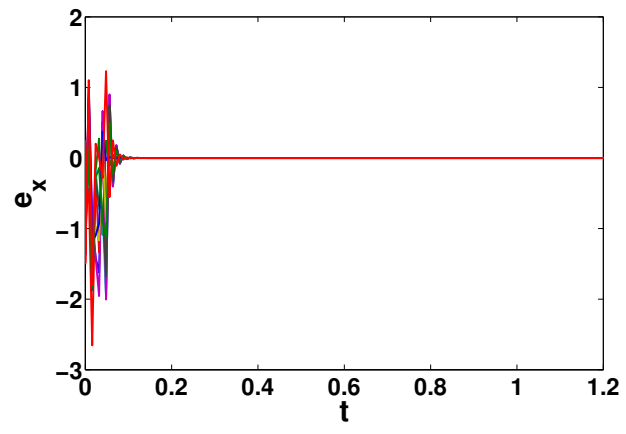

(b)

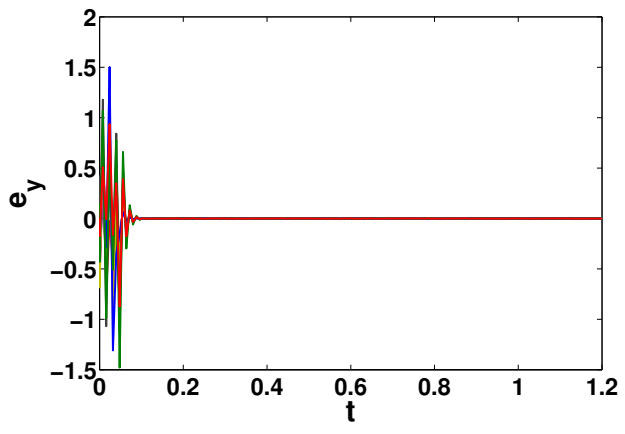

Figure 6: Dynamics of reduced-order HFPS errors with delay and non-delay coupling when $A=B$ : (a) errors of $x$ components; (b) errors of $y$ components. 


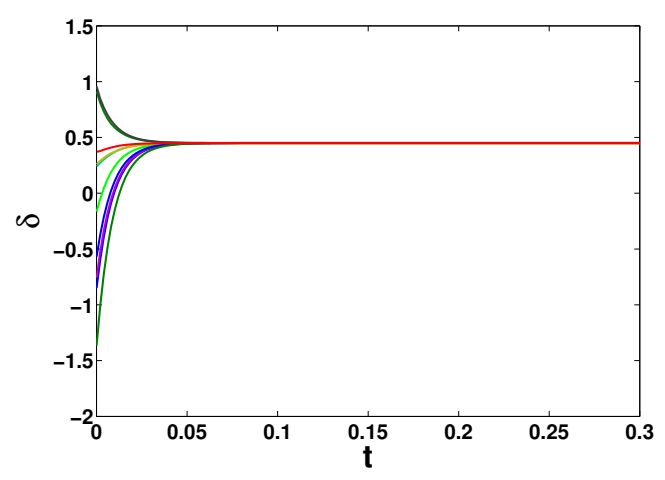

Figure 7: The estimated parameters $\hat{\delta}_{i}$ of adaptive feedback gains (3.6) in reduced-order case.

(a)

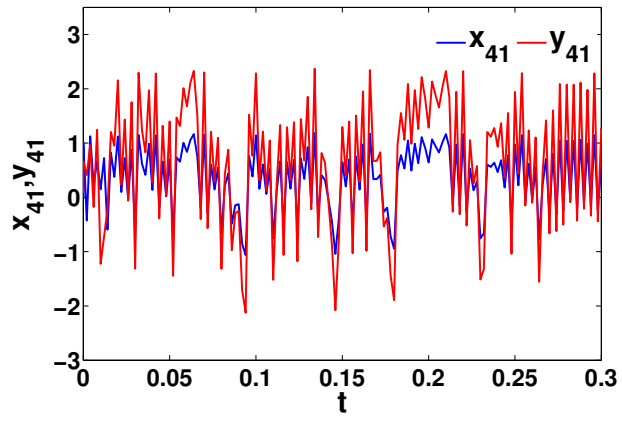

(b)

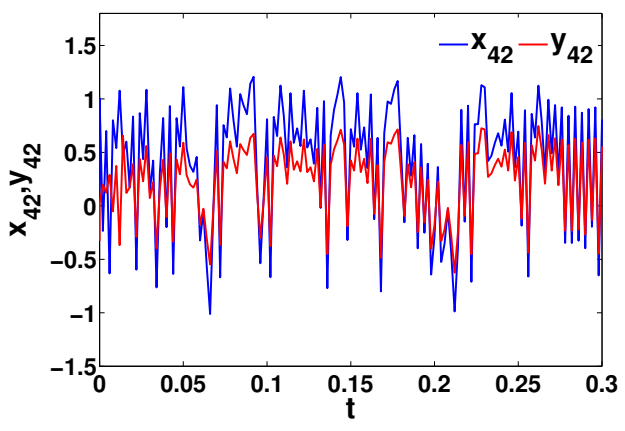

Figure 8: The time evolution of the variables of the 4 th nodes of networks with delay and non-delay coupling when $A=B$.

\subsubsection{The drive is signal system $(\mathrm{A}=0)$}

The numerical results are presented in Figures 9-11. The time evolution of the synchronization errors is illustrated in Figure 9, which shows that upon the activation of the control functions at $t=0.01$. The identified parameters of the adaptive gains are depicted in Figure 10 which converge to the fixed values $\delta_{i}$, which shows that the adaptive law designed is effective. These results prove that the required synchronization has been achieved with our designed control law (3.7)-(3.8) and under the conditions (3.3), (3.4). Figure 11 depicts the state trajectories between the drive system and the last five nodes in response networks.

(a)

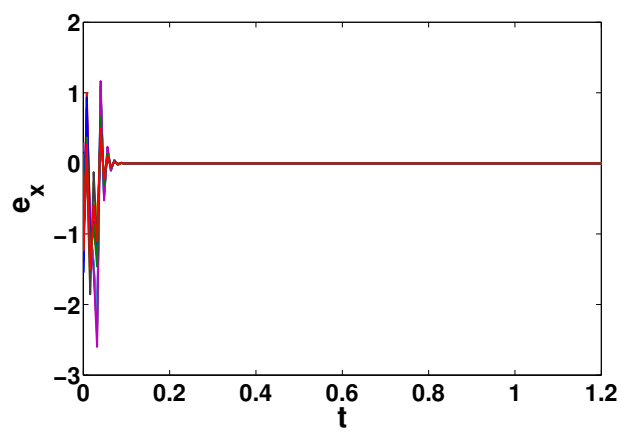

(b)

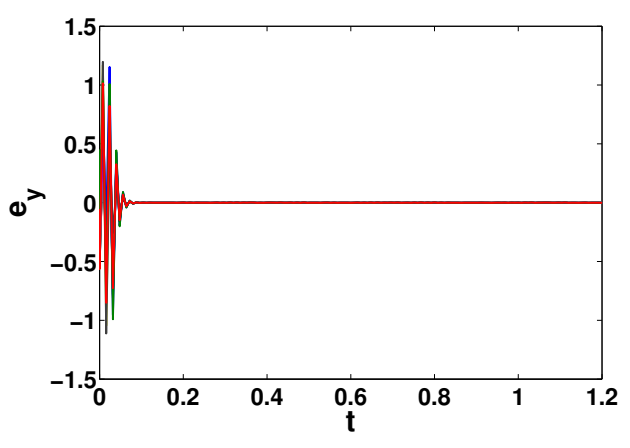

Figure 9: Dynamics of reduced-order HFPS errors with delay and non-delay coupling when $A=0$ : (a) errors of $x$ components; (b) errors of $y$ components. 


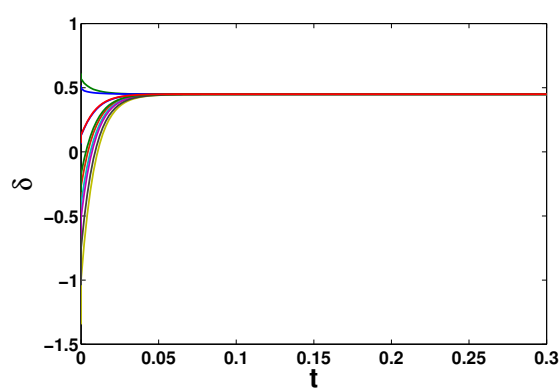

Figure 10: The estimated parameters $\hat{\delta}_{i}$ of adaptive feedback gains (3.8) in reduced-order case.

(a)

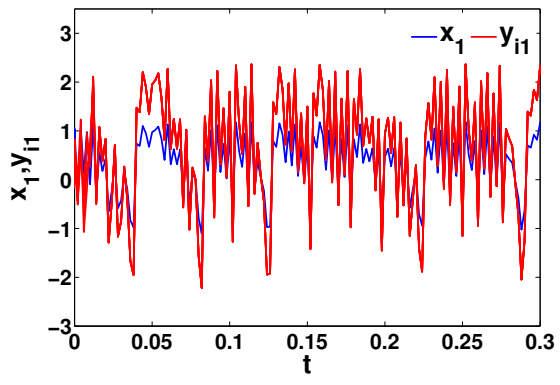

(b)

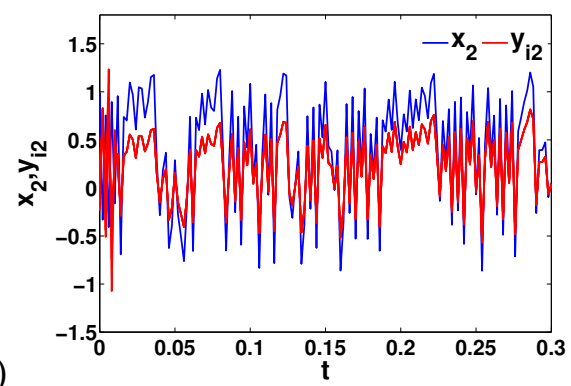

Figure 11: The time evolution of the variables between the drive system and the last five nodes of networks with delay and non-delay coupling when $A=0$.

\subsection{Case II: Increased-order synchronization when $\mathrm{m}<\mathrm{n}$}

In this subsection, we discuss the simulation results of the hybrid function projective synchronization when the order of the drive nodes are less than the order of the response nodes.

We will use the 2D Lozi map (4.3) as the drive network with 10 nodes. For the response network, the first 5 nodes are described by Hénon-like map (4.1) and the other 5 nodes are described by the following 3D generalized Hénon map (4.4):

$$
\begin{aligned}
& y_{i 1}(k+1)=-\beta y_{i 2}(k), \\
& y_{i 2}(k+1)=1+y_{i 3}^{2}(k)-\alpha y_{i 2}^{2}(k), \\
& y_{i 3}(k+1)=\beta y_{i 2}(k)+y_{i 1}(k),
\end{aligned}
$$

where $\alpha=1.07, \beta=0.3$.

From the bifurcation diagrams of the chaotic systems which are shown in Figure 1(a), (c), and Figure 12 , we can see that the evolution region of the network error $\left|e_{i}(k)\right|$ is $(0,1.8)$. We take the coefficient value is $\alpha_{i}>1.8$, where the function $\mathrm{Q}(\mathrm{k})$ satisfies the condition (3.4).

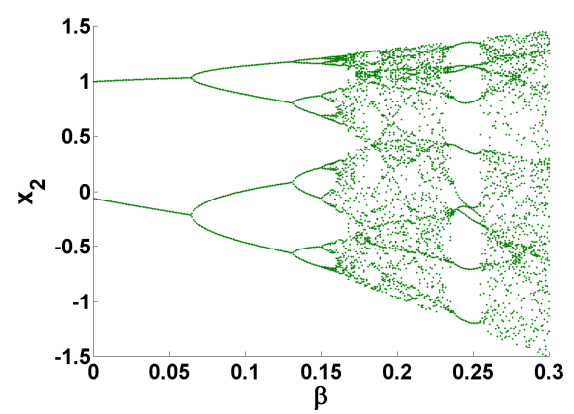

Figure 12: Bifurcation diagram of generalized Hénon map. 
In these numerical simulations, the initial condition of each node is chosen randomly. The outer coupling configuration matrices $A=\left(a_{i j}\right)$ and $B=\left(b_{i j}\right)$ are chosen to be as the same in the previous case. The feedback control gain is $\delta_{i}=0.6, \alpha_{i}=3$ and $\left.H=\operatorname{diag}[2 \cos (2 \pi k / 30)), 0.5+\sin (\pi \mathrm{k} / 5), 0.3 \cos (\pi \mathrm{k} / 30)\right]$.

\subsubsection{The networks have different topological structures $(A \neq B)$}

The numerical results are presented in Figures 13-15. The time evolution of the synchronization errors is depicted in Figure 13, which shows that upon the activation of the control functions at $t=0.01$. The estimation values of uncertain feedback gains $\hat{\delta}_{i}$ are depicted in Figure 14, which approach to the fixed value $\delta_{i}$ and shows that the identification law is effective. These results prove that the required synchronization has been achieved with our designed control law (3.1)-(3.2) and under the conditions (3.3), (3.4). Figure 15 depicts the state trajectories between the 2nd nodes of networks.

(a)

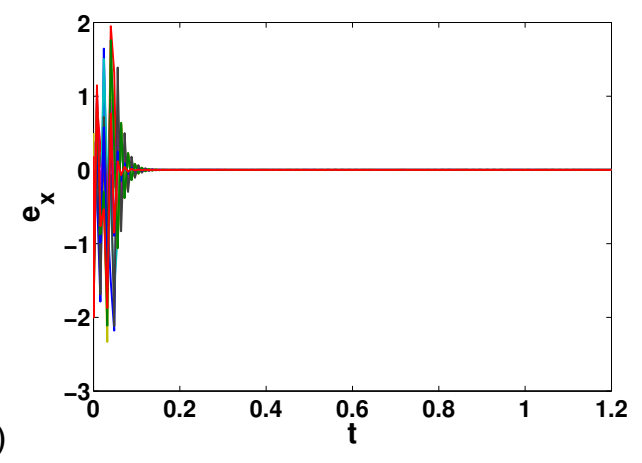

(b)

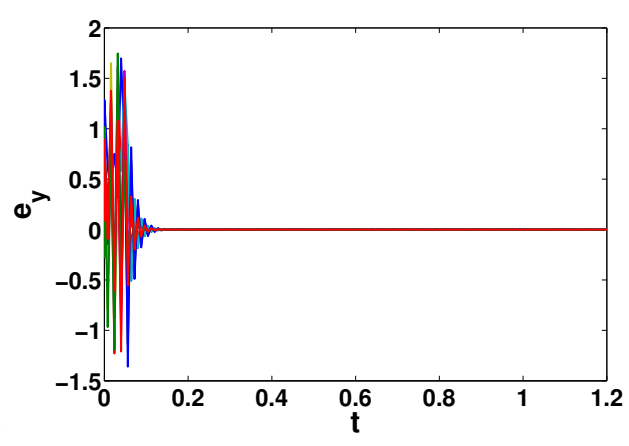

(c)

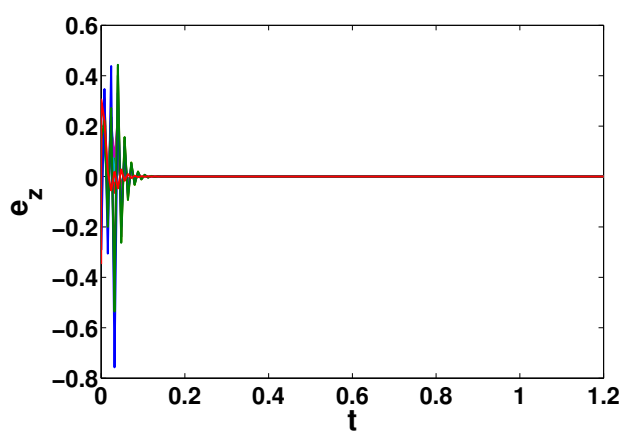

Figure 13: Dynamics of increased-order HFPS errors with delay and non-delay coupling when $A \neq B$ : (a) errors of $x$ components; (b) errors of $y$ components; (c) errors of $z$ components.

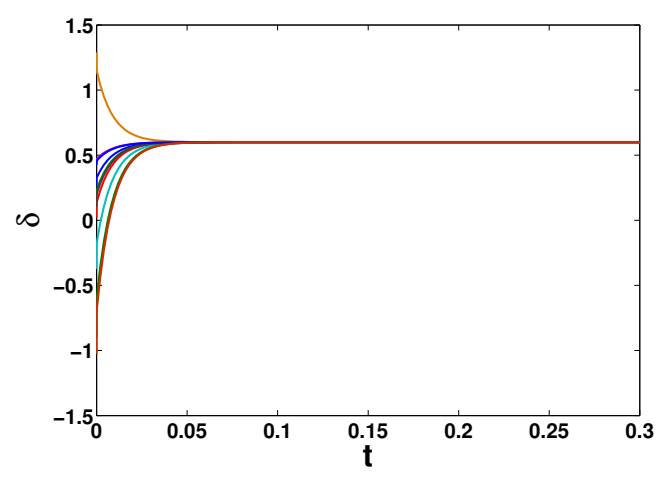

Figure 14: The estimated parameters $\hat{\delta}_{i}$ of adaptive feedback gains (3.2) in increased-order case. 
(a)

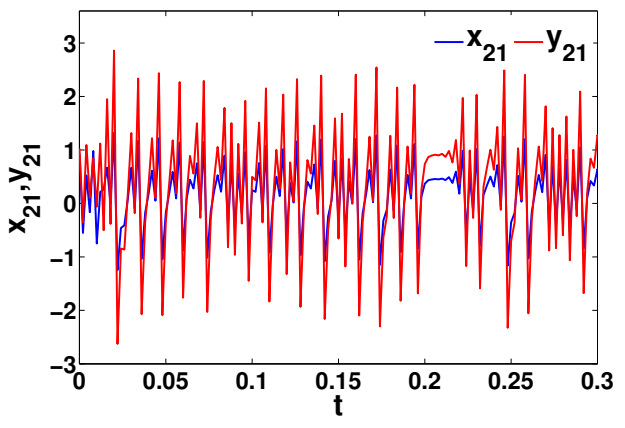

(b)

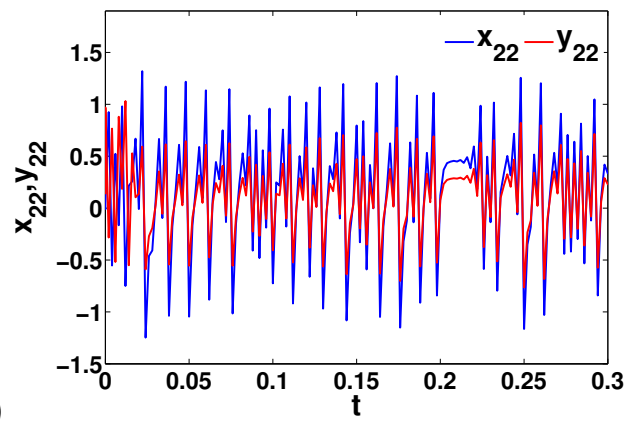

(c)

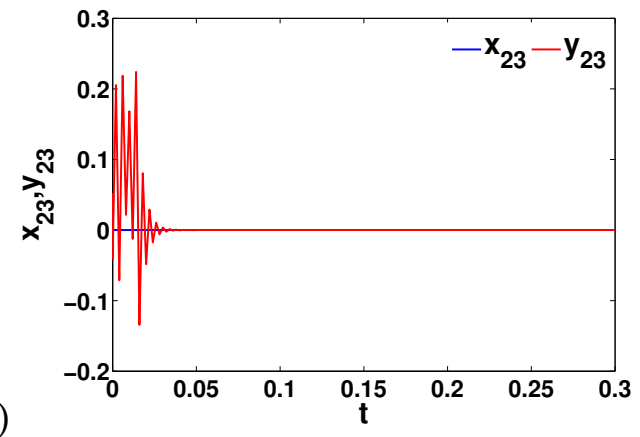

Figure 15: The time evolution of the variables of the 2 nd nodes of networks with delay and non-delay coupling when $A \neq B$.

\subsubsection{The networks have identical topological structure $(\mathrm{A}=\mathrm{B})$}

In this numerical results, Figure 16 shows all the error components approach to zero and therefore the error dynamics system reaches to the stability. The identified parameters of the adaptive gain are depicted in Figure 17 which converge to the fixed values $\delta_{i}$ and shows the effectiveness of our identification law. Figure 18 depicts the state trajectories between the 8 th nodes of networks.

(a)

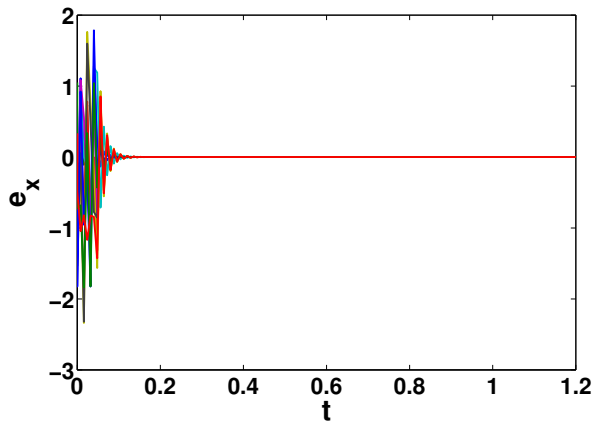

(b)

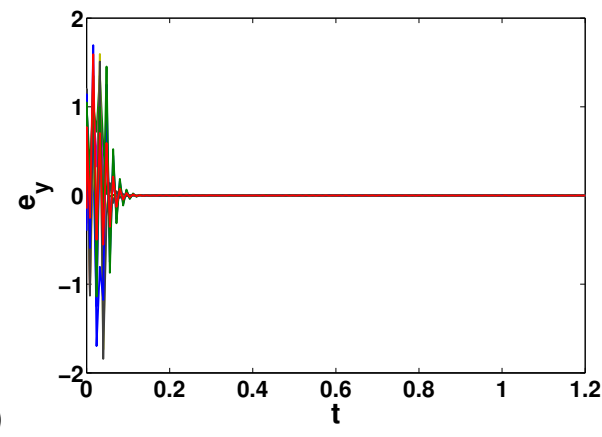

(c)

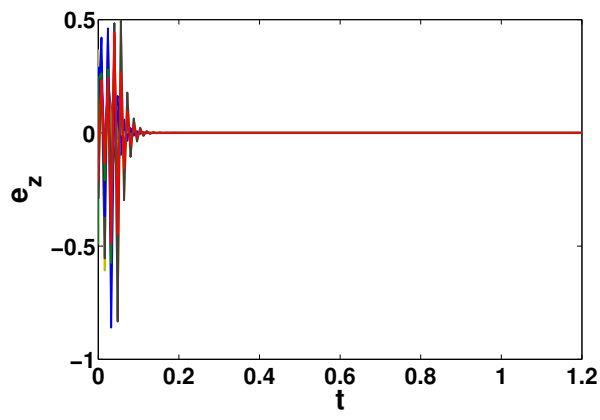

Figure 16: Dynamics of increased-order HFPS errors with delay and non-delay coupling when $A \neq B$ : (a) errors of $x$ components; (b) errors of $y$ components; (c) errors of $z$ components. 


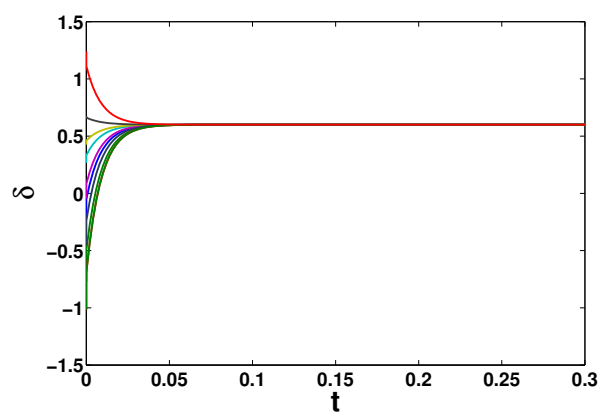

Figure 17: The estimated parameters $\hat{\delta}_{i}$ of adaptive feedback gains (3.6) in increased-order case.

(a)

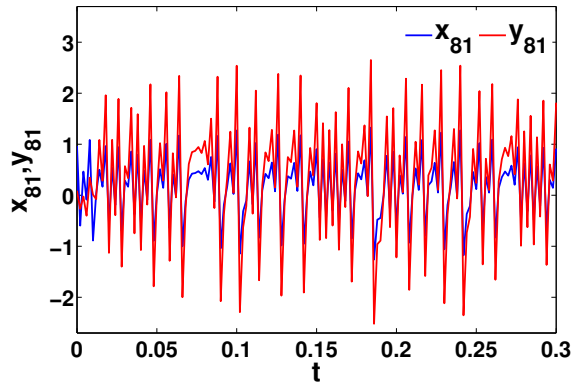

(b)

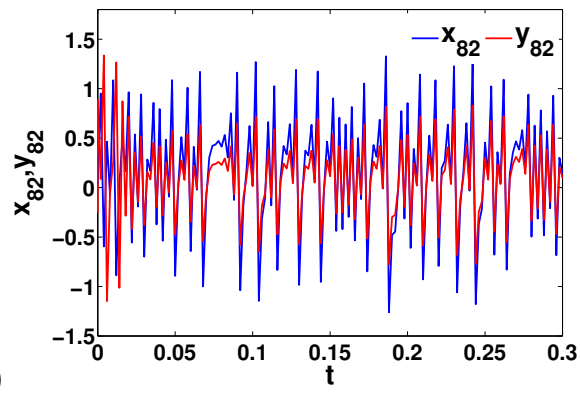

(c)

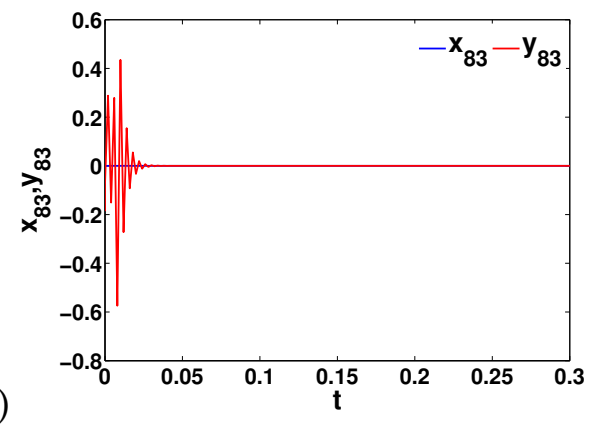

Figure 18: The time evolution of the variables of the 8th nodes of networks with delay and non-delay coupling when $\mathrm{A}=\mathrm{B}$.

\subsubsection{The drive is single system $(A=0)$}

The numerical results are presented in Figures 19-21. The time evolution of the synchronization errors is illustrated in Figure 19. The identified parameters of the adaptive gain are depicted in Figure 20 which converge to the fixed values $\delta_{i}$. These results prove that the required synchronization has been achieved with our designed control law (3.7)-(3.8) and under the conditions (3.3), (3.4). The state trajectories between the drive system and the last five nodes in response networks are depicted in Figure 21.

(a)

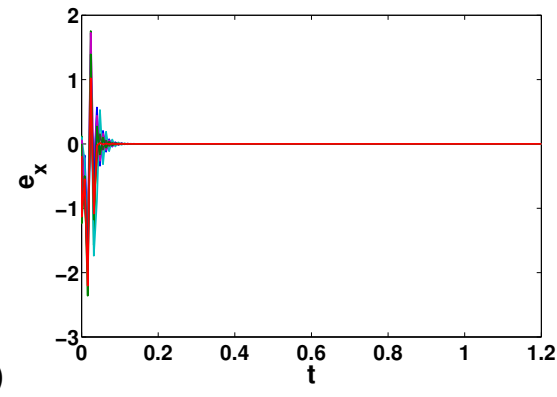

(b)

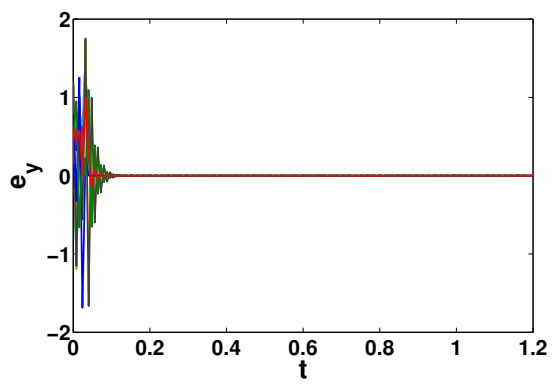


(c)

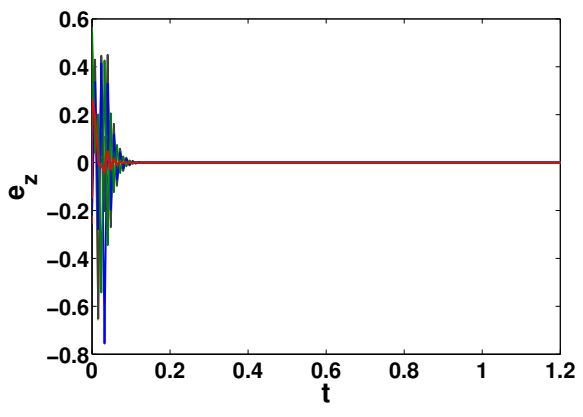

Figure 19: Dynamics of increased-order HFPS errors with delay and non-delay coupling when $A=0$ : (a) errors of $x$ components; (b) errors of $y$ components; (c) errors of $z$ components.

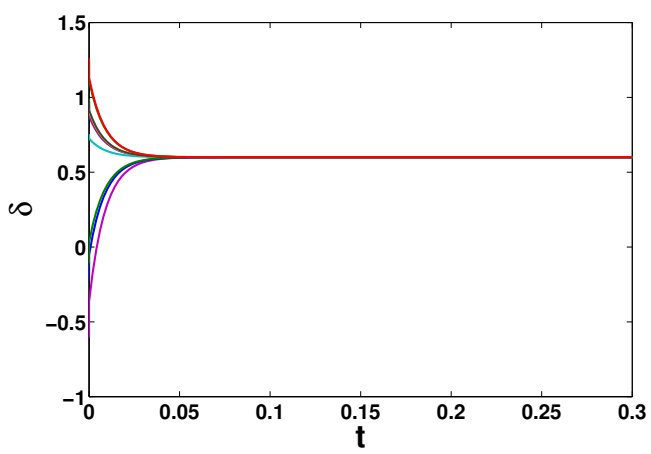

Figure 20: The estimated parameters $\hat{\delta}_{i}$ of adaptive feedback gains (3.8) in increased-order case.

(a)

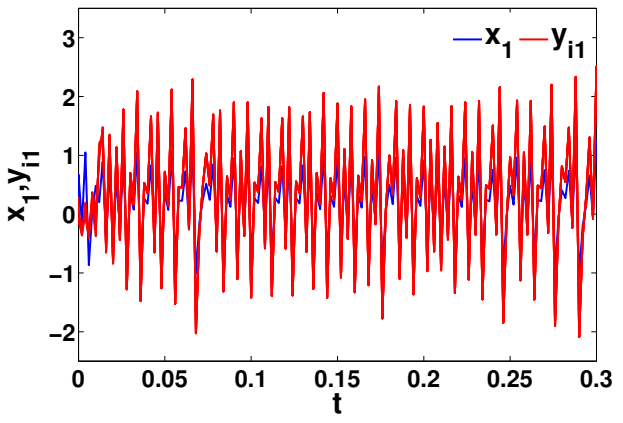

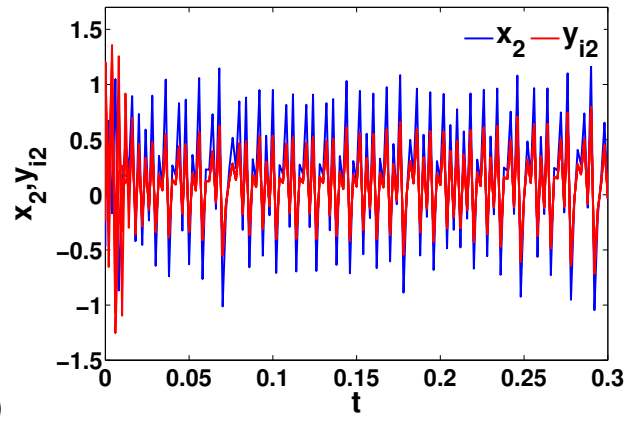

(b)

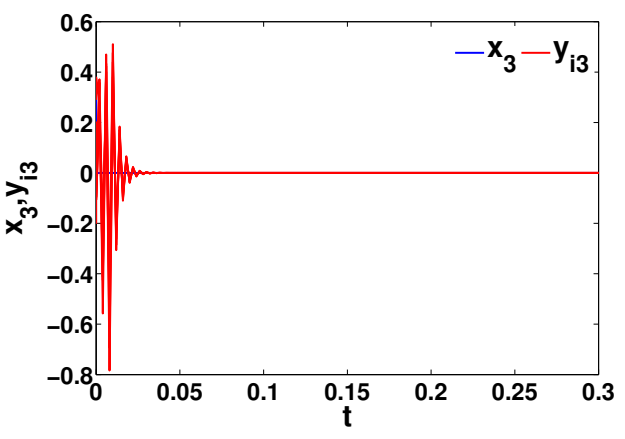

Figure 21: The time evolution between the drive system and the variables of the last five nodes of networks with delay and non-delay coupling when $A=0$. 


\section{Conclusion}

Hybrid function projective synchronization (HFPS) behavior of uncertain discrete complex dynamical networks (DCDNs) model with different orders is investigated. Based on Lyapunov stability theory and an adaptive control scheme, sufficient conditions were derived to achieve the hybrid function projective synchronization with different nodes and different orders. On the basis of the adaptive update law and the adaptive control gains were obtained. Furthermore, different cases of outer coupling matrix of the drive dynamics were considered. The simulation results revealed that the states of the dynamical network with non-delay and delay coupling could be asymptotically synchronized into a desired scaling function matrix under the designed controller. Finally, the numerical results proved the validity of the proposed method.

\section{Acknowledgment}

The authors would like to acknowledge the UKM research grant DIP-2017-011 and Ministry of Education, Malaysia grant FRGS/1/2017/STG06/UKM/01/1 for financial support.

\section{References}

[1] G. Al-mahbashi, M. S. M. Noorani, S. A. Bakar, Projective lag synchronization in drive-response dynamical networks with delay coupling via hybrid feedback control, Nonlinear Dynam., 82 (2015), 1569-1579. 1

[2] G. Al-mahbashi, M. S. M. Noorani, S. A. Bakar, Hybrid function projective synchronization of uncertain discrete complex dynamical networks, Int. J. Dynam. Control, 2016 (2016), 9 pages. 1

[3] M. M. Al-sawalha, M. S. M. Noorani, Adaptive increasing-order synchronization and anti-synchronization of chaotic systems with uncertain parameters, Chin. Phys. Lett., 28 (2011), 3 pages. 1

[4] M. M. Al-sawalha, M. S. M. Noorani, Chaos reduced-order anti-synchronization of chaotic systems with fully unknown parameters, Commun. Nonlinear Sci. Numer. Simul., 17 (2012), 1908-1920. 1

[5] R. Albert, A. L. Barabási, Statistical mechanics of complex networks, Rev. Modern Phys., 74 (2002), 47-97. 1

[6] S. Boccaletti, V. Latora, Y. Moreno, M. Chavez, D.-U. Hwang, Complex networks: structure and dynamics, Phys. Rep., 424 (2006), 175-308. 1

[7] H.-Y. Du, Function projective synchronization in drive-response dynamical networks with non-identical nodes, Chaos Solitons Fractals, 44 (2011), 510-514. 1

[8] X. Jian, Anti-synchronization of complex delayed dynamical networks through feedback control, Sci. Res. Essays, 6 (2011), 552-558. 1

[9] C.-P. Li, C.-X. Xu, W.-G. Sun, J. Xu, J. Kurths, Outer synchronization of coupled discrete-time networks, Chaos, 19 (2009), 7 pages. 1

[10] X.-W. Liu, T.-P. Chen, Synchronization analysis for nonlinearly-coupled complex networks with an asymmetrical coupling matrix, Phys. A, 387 (2008), 4429-4439. 1

[11] H. Liu, S.-G. Li, H.-X. Wang, Y.-H. Huo, J.-H. Luo, Adaptive synchronization for a class of uncertain fractional-order neural networks, Entropy, 17 (2015), 7185-7200. 1

[12] H.-M. Liu, W.-G. Sun, G. Al-mahbashi, Parameter identification based on lag synchronization via hybrid feedback control in uncertain drive-response dynamical networks, Adv. Difference Equ., 2017 (2017), 11 pages.

[13] A. Ouannas, M. M. Al-sawalha, Synchronization between different dimensional chaotic systems using two scaling matrices, Optik, 127 (2016), 959-963. 1

[14] S. A. Pandit, R. E. Amritkar, Characterization and control of small-world networks, Phys. Rev. E, 60 (1999), 15 pages. 1

[15] S. S. Strogatz, Exploring complex networks, Nature, 410 (2001), 268-276. 1

[16] W.-G. Sun, R. Wang, W.-X. Wang, J.-T. Cao, Analyzing inner and outer synchronization between two coupled discretetime networks with time delays, Cogn. Neurodyn., 4 (2010), 225-231. 1

[17] W.-G. Sun, S. Wang, G.-H. Wang, Y.-Q. Wu, Lag synchronization via pinning control between two coupled networks, Nonlinear Dynam., 79 (2015), 2659-2666. 1

[18] Z.-Y. Wu, Synchronization of discrete dynamical networks with non-delayed and delayed coupling, Appl. Math. Comput., 260 (2015), 57-62. 1

[19] B.-G. Xin, Z.-H. Wu, Projective synchronization of chaotic discrete dynamical systems via linear state error feedback control, Entropy, 17 (2015), 2677-2687. 1

[20] Z.-L. Yuan, Z.-Y. Xu, L.-X. Guo, Generalized synchronization of two bidirectionally coupled discrete dynamical systems, Commun. Nonlinear Sci. Numer. Simul., 17 (2012), 992-1002. 1

[21] Q.-J. Zhang, J.-A. Lu, J.-C. Zhao, Impulsive synchronization of general continuous and discrete-time complex dynamical networks, Commun. Nonlinear Sci. Numer. Simul., 15 (2010), 1063-1070. 1

[22] X.-B. Zhou, L.-L. Xiong, X.-M. Cai, Adaptive switched generalized function projective synchronization between two hyperchaotic systems with unknown parameters, Entropy 16 (2013), 377-388. 1 